\title{
The perks of going tobacco free: A Pioneer experience in a Portuguese Acute Inpatient Psychiatry Unit
}

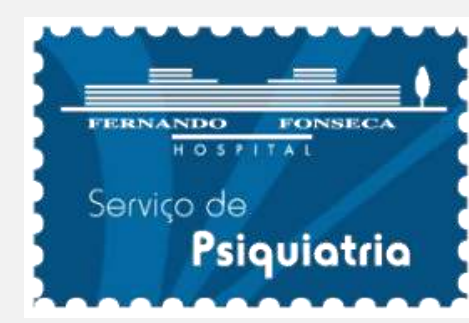

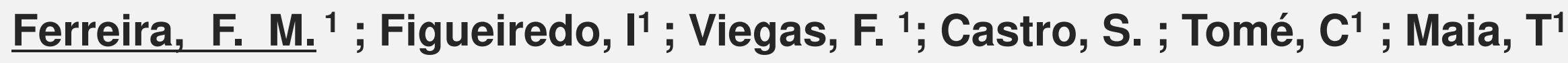 \\ ${ }^{1}$ Department of Psychiatry - Hospital Prof. Dr. Fernando Fonseca, Amadora, Portugal
}

\section{BACKGROUND AND AIMS}

In the last few decades, important public health policies promoting an environment free of tobacco have been taken in place in Portugal and in international scenarios. This represented a wave of change in social paradigms, contributing to the reduction of the prevalence of smoking in general population. Under the Portuguese National Health Plan, several measures are identified in order to reduce tobacco use, contemplating, among others, the protection of non-smokers from exposure to environmental tobacco smoke.

The prevalence of cigarette smoking in people with serious mental illnesses (SMI) is much higher than in the general population. Tobacco smoking is a major contributor to high premature mortality and severe morbidity in people with SMI.

The culture of smoking by patients and staff within mental health systems has been a longstanding and accepted cultural norm. This paradigm has been challenged in the past few years, with the successfully introduction of smoke-free policies in mental health settings in many countries.

Literature suggests that treatment interventions based on behavioral support and pharmacotherapy applied in general population are also effective in patients with SMI. Moreover, recent reviews indicate that total smoking bans in psychiatric settings are not associated with the increase of the incidence of physical assaults or disruptive behaviors.

Nonetheless, in Portugal, according to the of Decree Law n. ${ }^{0}$ 37/2007 of 14th August, mental health facilities constitute one exception to the total ban adopted in health-care settings, allowing patients to smoke in designated areas, which contributes to maintenance of a smoking paradigm towards psychiatric patients.

In this poster we aim to present the process of implementation of a comprehensive smoke-free policy in an Acute Inpatient Psychiatry Unit, for the first time in Portugal.

\section{MATERIAL AND METHODS}

Non-systematic literature search was conducted, throughout electronic databases (PubMed, Google Scholar, Elseviver and Cochrane). Search terms combinations with derivatives of "mental health", "psychiatric unit" and "smokefree policies", "smoking ban" were used. No restrictions were made on date or country of publication.

Description of the projec implementation process.

\section{RESULTS AND DISCUSION}

\section{BEFORE IMPLEMENTATION}

STAGE 0: Non-systematic literature search was conducted, throughout electronic databases to access evidence regarding smoke free policies in psychiatric wards.

Additional information was collect, namely from hospital internal protocols from other medical areas, related to smoke free intra-hospital policies.

Conception of an internal protocol: using a multidisciplinary and systemic approach, involving multiple staff entities and in cohesion with hospital policies.

STAGE 1: A comprehensive smoke-free policy across the acute inpatient unit started from $16^{\text {th }}$ October- $16^{\text {th }}$ December 2018. Preparations for this began 6 months earlier, with scheduling and definition of the program goals and steps.

3 sessions for staff training took place, with collaboration with Ligation Psychiatry team and hospital-based specialists from smoking cessation team.

Staff training and cohesion team strategies were conducted during all the process

\section{IMPLEMENTATION}

The smoke-free policy included the prohibition of smoking in the buildings and grounds of all hospital premises, no staff facilitated smoking, and a tobacco dependence treatment pathway.

The day before the beginning of the project ( $15^{\text {th }}$ October 2018),

information and counseling of the patients already admitted in the unit was offered

At admission, tobacco use was accessed by the nursing team.

Treatment were offered to smokers, and included nicotine replacement therapy (NRT) within $30 \mathrm{~min}$ of arrival on the ward and brief interventions with the staff to manage ttemporary abstinence symptoms.

During the inpatient period, patients were offered NRT, counselling, and brief interventions regarding smoking cessation. Information was offered to the families as well.

Occupational Therapy activities, focusing healthier life habits took place. At the first community appointment smoking status was accessed and cessation support was also provided at discharge and on return to the community.

When important medical co-morbidities existed (e.g. DPOC or cardiovascular problems) patients were referred to hospital-based specialist smoking cessation interventions.

EVALUATION:

- Two months before (August and September 2018) and during the implementation of the program data regarding variables as occurrence of violent events and violations to the ban were collect, in order to access the program viability.

\section{PRELIMINARY RESULTS}

No increase in physical events after the total ban.

Most frequent incidents registered were related to violations of the ban. PROJECT STATUS:

$\checkmark$ The project has been implemented and is sustained. Data analysis is still ongoing.

$\checkmark$ Community impact of the smoke-free program in the acute unit as yet to be evaluate

\section{CONCLUDING REMARKS}

The integration of smoking-related issues in mental health care pathways appears paramount Greater awareness needs to be raised among mental health providers, in order to acknowledge tobacco use as a vital sign in mental healthcare provision.

This project, pioneer in Portugal, demonstrates the need for tobacco reconceptualization in psychiatry, with clear impact in reducing the health inequalities and stigma surrounding this patients.

\section{Clinical implications}

- This project shows that, with adequate preparation, it is possible to implement a total smoking ban in a acute psychiatric setting without serious negative consequences, in line with previous literature.

- Team extensive preparation and coherence, with clear timelines and actuation protocols definition appeared to be a crucial part of the success.

\section{REFERENCES}

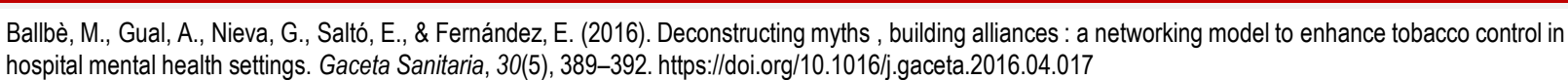

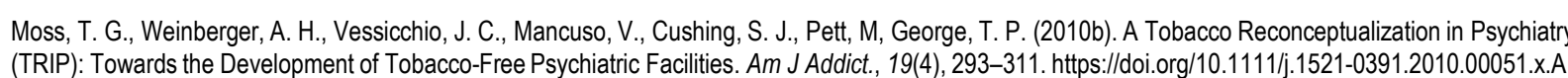

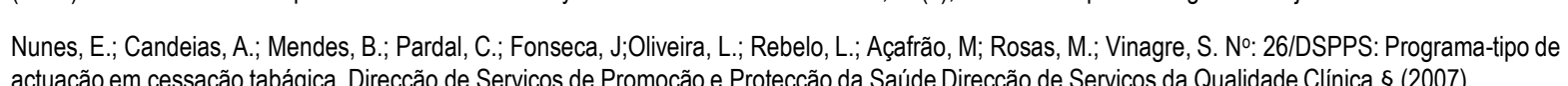

Robson, D., Spaducci, G., McNeill, A., Stewart, D., Craig, T. J. K., Yates, M., \& Szatkowski, L. (2017). Effect of implementation of a smoke-free policy on physical violence in a psychiatric

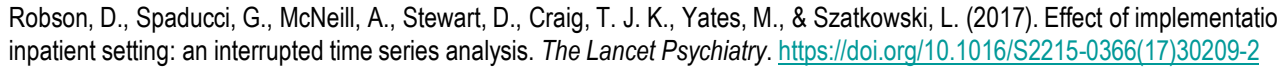

Rüther, T., Bobes, J., De Hert, M., Svensson, T. H., Mann, K., Batra, A., ... Möller, H. J. (2014). EPA Guidance on tobacco dependence and strategies for smoking cessation in people Sharma, R., Gartner, C. E. \& H Hall, W. D. (2016). The challenge of reducing smoking in people with serious mental illness. The Lancet Respiratory Medicine, 4(10), 835-844.
httrps://doiorg/10.1016/22213-2600(16)30228-4 